\title{
Highlights of Hinode Observations and Future Prospects
}

\author{
By Taro SAKAO ${ }^{1)}$ \\ ${ }^{1)}$ Institute of Space and Astronautical Science, JAXA, Sagamihara, Japan
}

(Received July 7th, 2009)

\begin{abstract}
The third Japanese solar-observing satellite, Hinode, has been revolutionalizing our understanding on magneto-plasma activities that prevail all layers of the solar atmosphere, namely, photosphere, chromosphere, transition region and corona. We present highlights of solar observations so far made with Hinode, and discuss possible future solar mission which has been under extensive investigation, based on Hinode results, among Japanese and international solar community.
\end{abstract}

Key Words: Sun, Plasmas, Magnetic Activity

\section{Introduction}

Solar activities such as explosive energy release in flares, massive ejections of coronal plasmas called CMEs (coronal mass ejections), and outflow of the solar wind into the interplanetary space, take place in magnetized atmosphere of the Sun. Since its launch in 2006, the third Japanese solar-observing satellite, Hinode ${ }^{1)}$, has continuously been providing us with ample findings about the Sun which were never thought of before. Hinode aims to investigate magneto-hydrodynamic coupling between the photospehre (visible surface of the Sun), where magnetic field lines in the solar atmosphere are rooted, and the corona, where dissipation of magnetic energy results in dynamics and heating of the plasmas there.

In order to pursue the above scientific objective, Hinode carries a suite of three state-of-the-art telescopes, Solar Optical Telescope $(\mathrm{SOT})^{2)}$, X-Ray Telescope (XRT) ${ }^{3,4)}$, and EUV Imaging Spectrometer (EIS) ${ }^{5}$. SOT employs Gregorian optics with 50-cm diameter primary mirror (Optical Telescope Assembly; OTA), and a focal-plane instrument package (FPP), and performs vector magnetic field measurements on the photosphere with diffraction-limited angular resolution of 0.2-0.3", together with photospheric/chromospheric imaging and Dopplergram measurements. XRT performs highresolution imaging (consistent with 1" focal-plane CCD pixel size, which is three times better than the Soft X-ray Telescope (SXT) aboard Yohkoh) and temperature diagnostics of coronal plasmas from below $1 \mathrm{MK}$ to above $10 \mathrm{MK}$ with grazing incidence optics. EIS carries out line-of-sight Doppler velocity measurements as well as temperature/density diagnostics for coronal plasmas with 3-10 times improved performance as compared to its predecessor (CDS instrument aboard SoHO mission). The three telescopes are mounted on a single optical bench made of CFRP (Optical Bench Unit; OBU) so as to maintain mutual coalignment. Figure 1 illustrates schematic view of the spacecraft.

All the three telescopes were developed, and have been operated, under very close international collaboration with NASA (U.S.), STFC (U.K.), and ESA (Europe). NASA

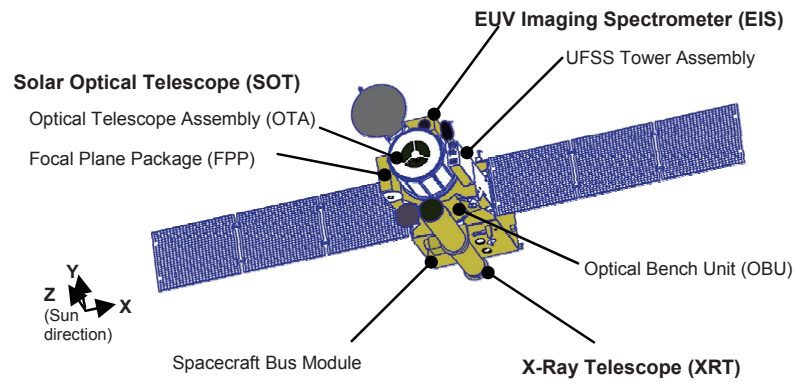

Fig. 1. A schematic illustration of Hinode with the three telescopes; SOT, XRT, and EIS.

participates in all the three instruments, STFC participates in EIS, and ESA performs extensive post-launch data downlink support.

\section{Hinode Highlights}

\subsection{Activities in the polar region}

The polar regions of the Sun had been considered to be inactive in X-rays (because of the presence of polar coronal holes) and weak in magnetic field strength (because of weak line-of-sight field strength) before Hinode. Observations of the polar regions with Hinode have revolutionized such conventional view. XRT discovered surprizingly-frequent ( $\sim 60$ events per day) occurrence of soft X-ray jets across the polar coronal holes ${ }^{6-8)}$. Meanwhile, SOT identified, in the polar region of the Sun (70-90 degrees in lattitude), concentration of strong vertical magnetic field forming magnetic patches each with size, say, 1,000-3,500 km, and magnetic field strength reaching as high as, or even exceeding, $1 \mathrm{kG}^{9)}$ (Figure 2). The kG-patches have the same magnetic polarity (either positive or negative) in the south (or north) polar region, with magnetic field lines from the patches mostly orthogonal (local inclination smaller than 25 degrees) to the solar surface.

The presence of strongly-concentrated field patches is 
expected to be responsible for accelerating the fast solar wind originated from the polar coronal hole regions of the $\operatorname{Sun}^{10,11)}$. Alfven waves propagating upwards in the solar atmosphere along magnetic field lines from the kG-patches may escape from being reflected back $^{12)}$ at the chromospheric boundary despite of the density decrease there due to rapid expansion (opening) of the field lines towards higher altitudes ${ }^{9}$. It may be possible that the propagating Alfven waves could eventually result in heating and acceleration of the fast solar wind by dissipating in the solar atmosphere, as has been indicated by theoretical investigations ${ }^{13)}$.

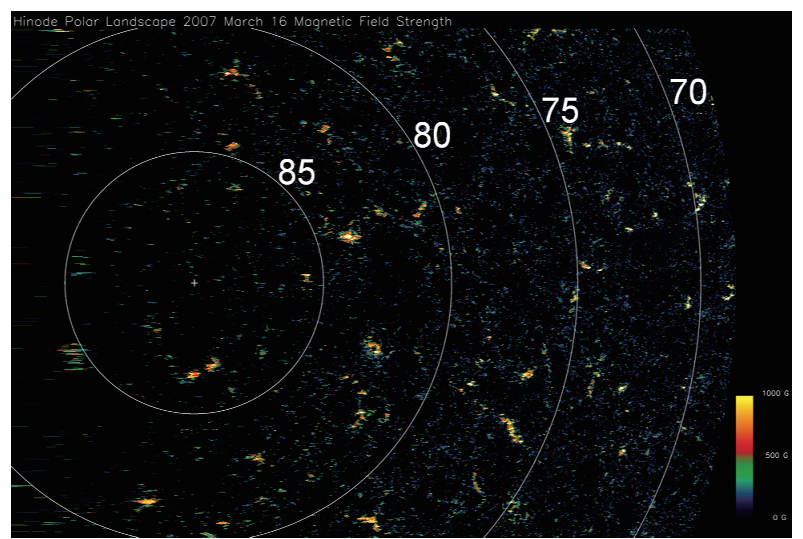

Fig. 2. Distribution of magnetic field strength in the south polar region observed by SOT aboard Hinode. Yellow in color indicates that the magnetic field strength reaches at around $1 \mathrm{kG}$. Numbers $(70,75,80$, and 85 ) in the figure indicate heliographic lattitudes in degrees. (After Tsuneta et al. ${ }^{9}$.)

Detailed comparison in locations between kG-patches seen with SOT and soft X-ray jets seen with XRT has revealed that the X-ray jets are most likely generated by interation (magnetic reconnection) between field lines from the kG-patches and small bipoles emerged in the vicinity of the kG-patches, or were transported from low-lattitude regions ${ }^{14)}$.

The discovery of strong magnetic field concentration and high X-ray activities in the polar region has invoked various interest addressed to the polar regions such as generation mechanism of concentrated strong magnetic field and its possible implications to the solar dynamo.

\subsection{The dynamic solar atmosphere}

Another significant finding of Hinode is that the solar atmosphere, in particular chromosphere which is the interface layer between the photosphere and the corona, is rich in dynamic activities such as jets which was not known before ${ }^{15)}$.

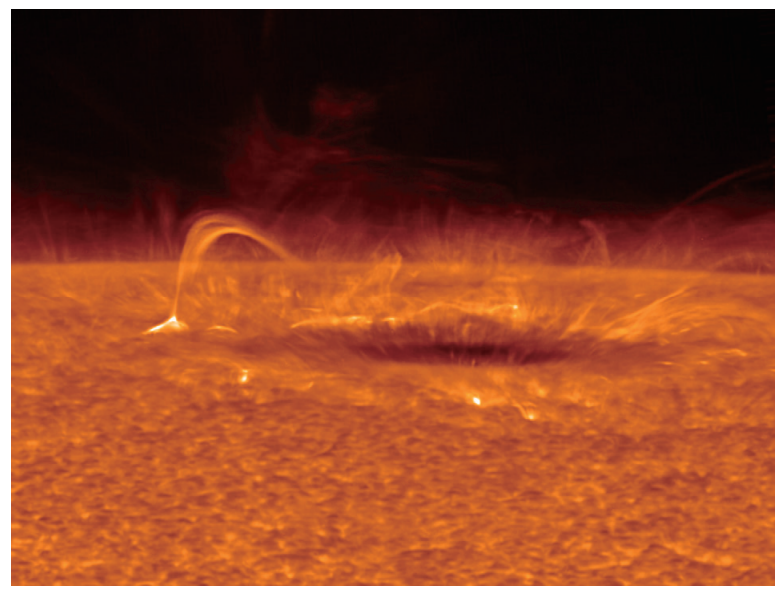

Fig. 3. A chromospheric jet observed with SOT in the Ca II H wavelength, in the vicinity of a sunspot located near the solar limb.

Figure 3 depicts an example of such activities where a jet event is seen in the chromsphere (chromospheric jet) in the $\mathrm{Ca}$ II H wavelength band (3968.5 A) of SOT. Observed shape, hence possibly magnetic configuration, of the chromospheric jets closely resembles that of coronal X-ray jets (i.e., 'inverted Y-shape'). This strongly suggests that magnetic reconnection process, which is responsible for the formation of jets, takes place throughout the chromosphere, at a much smaller scale (even smaller than 1 arcsec, or several hundred kilo-meters) as compared to coronal X-ray jets. An important feature regarding chromospheric jets is that their reaching-height (typically 2,000-5,000 km) cannot be accounted for by simple ballistic motion whose resultant height would be at most $\sim 200$ $\mathrm{km}$ with the observed jet velocity of $10-20 \mathrm{~km} / \mathrm{s}$. This may

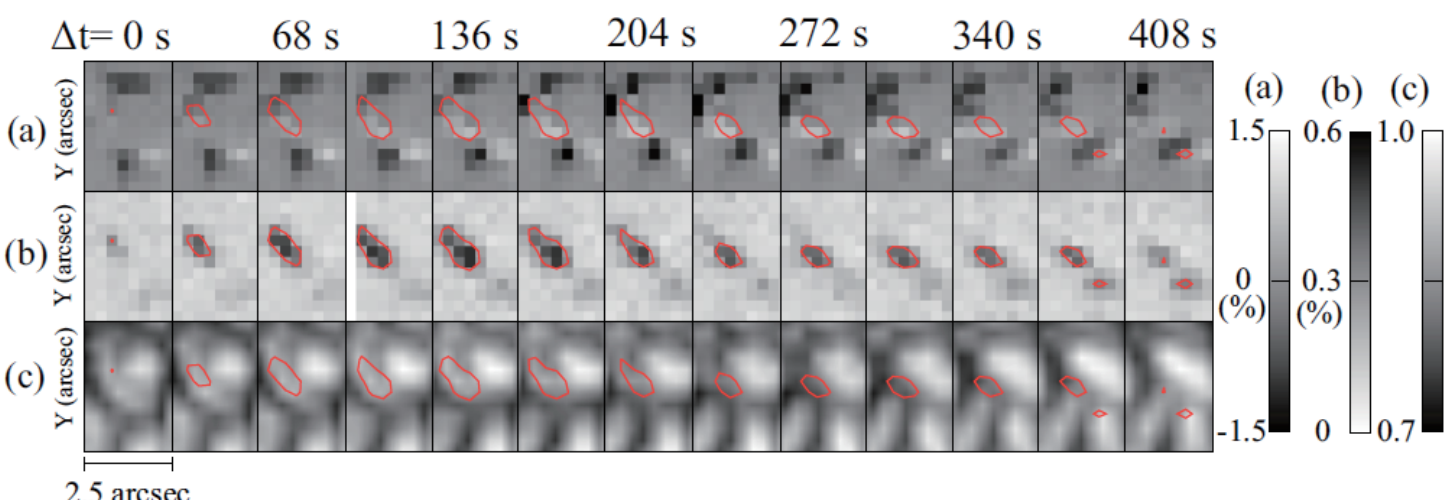

Fig. 4. An example of emergence and disappearance of transient horizontal magnetic field. Row (a): Indicates magnetic field strength vertical to the local solar surface. Row (b): Indicates magnetic field strength parallel to the local surface. Row (c): Intensity. Time goes from left to right in each row, covering a duration of 408 seconds. The feature referred to as 'transient horizontal magnetic field' is shown by red circles, indicating that the lifetime of the horizontal field is $\sim 6$ minutes. (After Ishikawa et al. ${ }^{16)}$ ) 
indicate possible presence of mechanism(s) other than the initial material ejection at the footpoints of jets, forming the main body of the jets, probably with magneto-hydrodynamic waves propagating along magnetic field lines ${ }^{15)}$.

The precise vector magnetic field measurement capability of SOT has revealed ubiquitous presence of 'horizontal magnetic field' across the surface of the Sun ${ }^{16,17)}$. It was found there is short-lived (lifetime typically 6 minutes), small-scale (maximum size identified to be $\sim 1 " .4 \times 1 "$ ) magnetic field whose direction parallel to the solar surface (hence called horizontal magnetic field) everywhere in plage regions and in the quiet Sun. Each transient horizontal field appear and disappear inside a granulation cell. This observation suggests that the generation of transient horizontal field is associated with convective motion of granules, possibly by some local dynamo process. In addition to the fact that this horizontal field is a new form of magnetic distribution on the photosphere that was not conceived before Hinode, magnetic energy flux brought in by the horizontal field is estimated to be comparable to that needed for heating the chromosphere, and sufficient for heating the corona ${ }^{17)}$. The newly-discovered transient horizontal magnetic field might be an essential agent for heating the chromosphere and the corona. Theoretical investigation on the possibility of heating the upper atmosphere with the transient horizontal field is also under way $^{18)}$.

\section{Future Prospects}

Based on the discoveries so far made with Hinode, two plans has been extensively investigated among Japanese solar physics community for the next Japanese solar mission, with close discussion with U.S. and European scientists. One is to seek for the possibility of exploring polar regions of the Sun (referred to as 'Plan $\mathrm{A}^{\prime}$ ) and the other to investigate the origin of dynamism in every layer of the atmosphere by performing spectro-polarimetric measurements ('Plan B'). As was the case for Yohkoh and Hinode, close international collaboration is conceived for the next mission, but even more extensively, with U.S. and European colleagues.

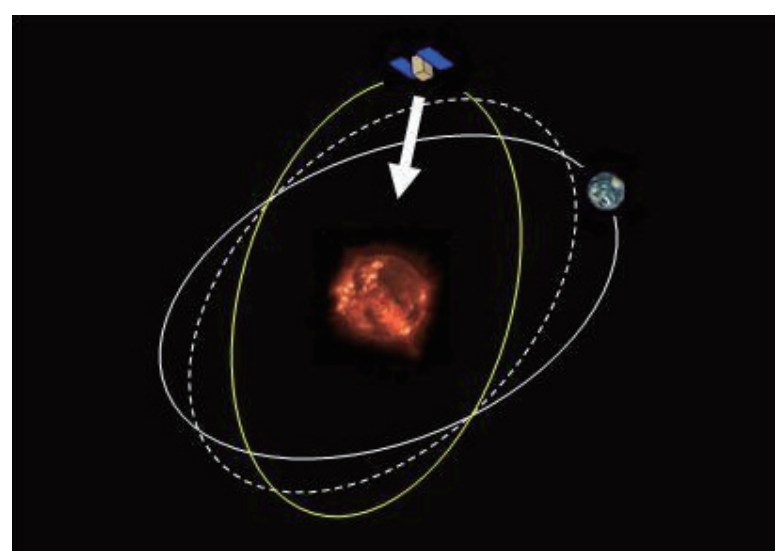

Fig. 5. Concept of out-of-ecliptic observation of the Sun with Plan A spacecraft (ion engine option case).

\subsection{Polar investigation}

Plan A aims to conduct out-of-ecliptic magnetic, X-ray and helioseismic observations of the polar and the equatorial regions (Figure 5) through which properties of the polar region and magnetic structure inside the Sun will be investigated. Apart from in-situ measurements carried out by Ulysses, polar regions of the Sun have never been explored. Investigation of the Sun's polar regions may shed some light on the origin of the fast solar wind which is believed to come from the polar regions. Also, lack of precise helioseismic measurements on the high lattitude regions of the Sun has so far been prevending any reliable estimate on the internal strucutre of the Sun at high lattitudes.

The Plan A spacecraft is going to carry a suite of payloads whose total mass probably at around $100 \mathrm{~kg}$ while the initial (wet) spacecraft mass $\sim 1,300 \mathrm{~kg}$. Preliminary model payloads include: a visible-light imager for helioseismic and magnetic measurements, and an X-ray/EUV coronal imager for investigating the polar corona. Several orbit maneuver options are under study, including (a1) increase orbit inclination by ion engines while maintaing Sun-Earth distance at $1 \mathrm{AU},(\mathrm{a} 2)$ increase orbit inclination by ion engines, with the aid of Earth swing-by, and (b) Jupiter and Earth swing-by without ion engines. In the case of (a2) for instance, the spacecraft is designed to reach the inclination angle of 30 degrees 3 years after the launch while 45 degrees 6 years.

\subsection{Dynamism of the magnetized atmospheric layers}

Plan B pursues high spatial resolution, high throughput (hence high cadence) spectroscopic, and wherever possible, even polarimetic, observations on the entire layers of the solar atmosphere; from the photosphere, through the chromosphere, towards the corona (Fig. 6).

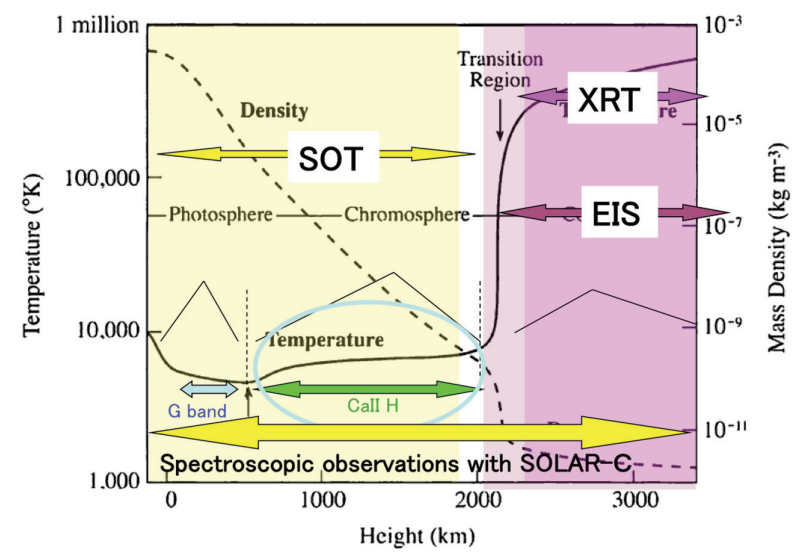

Fig. 6. Schematic illustration on the coverage of solar atmosphere to be made with the next solar mission (SOLAR-C) as compared to that made with SOT, XRT, and EIS aboard Hinode.

As Hinode lacked quantitative diagnostic capability (imaging-spectroscopic capability) for the activities discovered in the chromosphere, Plan B puts more weight on spectroscopy than Hinode which chiefly has its observational weight on imaging as far as chromosphere is concerned. Also, in order to adequately trace dynamic behavior of the 
chromosphere, Plan B observations have to be made with high cadence. For this purpose, Plan B instruments aim to perform, (1) in addition to the photosphere, chromospheric and, if possible, coronal spectro-polarimetry for vector magnetic field measurements, and (2) high spatial/temporal resolution spectroscopy for the dynamics in the chromosphere and the transition-region. With such capabilities, activities in each atmospheric layers and associated energy transfer through the atmosphere will be quantitatively investigated. Preliminary model payload for Plan B mission includes: a large-aperture telescope covering near-IR, visible, and UV wavelengths whose mirror diameter probably even larger than that of Hinode $(50 \mathrm{~cm} \phi)$, a high-resolution X-ray/EUV imager, and a high-resolution, high-throughput imaging spectrograph.

Two possibilities on the spacecraft orbit, geostationary orbit and Sun-synchronous polar orbit just like Hinode, have been under study from the viewpoint of large telemetry downlink rate (estimated to be even larger than $10 \mathrm{Mbps}$ ) required for Plan B.

\subsection{Status and schedule}

A working group to study the next solar mission was established under the ISAS/JAXA Space Sciences Steering Committee in December 2007 ('SOLAR-C Working Group'). Since then, in addition to science investigation on the two plans, various study activities have been ongoing which include system design study (ion engines and orbit design etc.) of Plan A spacecraft, and command and telemetry capabilities for Plan A and Plan B, in close collaborations with engineers from JAXA and industrial partners.

An international science definition meeting was held in November 2008 at ISAS where the latest science results relevant to Plan A/B and science significance of the two plans were extensively discussed among scientists from Japan, U.S. and Europe. It was agreeed among the participants that science with the two plans should be further investigated together with engineering feasibilities, before the selection for the plan is made. For this purpose, a total of five sub-working groups were formed under the master working group. These are: (1) Engineering investigation of Plan A spacecraft, (2) Helioseismology and the solar dynamo, (3) Chromosphere/ corona field measurements, (4) UV/EUV high-throughput spectrometry, and (5) Next generation X-ray telescope. Each sub-working group, except for the first one, consists of international participants and aims to clarify science and technical feasibility on the relevant subject.

The sub-working group activities are planned to continue for a year followed by compilation of a mission proposal which is to be submitted to JAXA in around 2010. In parallel to the mission proposal preparation, attempt to establish international framework for collaboration with NASA and ESA will be made. Regarding enginnering investigation on Plan A spacecraft, a working group preparation team was formed under JSPEC/JAXA Space Exploration Committee in January 2009 which takes lead in engineering aspects of Plan A feasibility study while science investigation conducted under initiative of ISAS/JAXA SOLAR-C working group. Projected launch would be around 2017.

\section{Summary and Conclusion}

Hinode has been providing ample new findings on magnetic activities in the solar atmosphere which are revolutionalizing our understanding on the Sun. These findings include frequent $\mathrm{X}$-ray jets and magnetic field concentration in the polar regions, unexpectedly-high jet activities in the chromosphere, and transient horizontal magnetic field ubiquitously covering the photosphere.

Based on such Hinode results, two possibilities on the next Japanese solar mission, Plan A and Plan B, are currently under investigation. Plan A is to conduct out-of-ecliptic observations of the polar regions for the first time since in-situ measurements by Ulysses. Plan B pursues high spatial resolution, and spectroscopic, observations seamlessly from the photosphere, chromosphere, towards the corona. A set of international sub-working groups is extensively studying science as well as technical feasibilities on several key aspects on Plan A and B. We attempt to realize the next mission shortly with international collaboration towards the projected launch in the latter half of 2010's.

\section{Acknowledgments}

Hinode is a Japanese mission developed and launched by ISAS/JAXA, collaborating with NAOJ as a domestic partner, NASA and STFC (UK) as international partners. Scientific operation of the Hinode mission is conducted by the Hinode science team organized at ISAS/JAXA. This team mainly consists of scientists from institutes in the partner countries. Support for the post-launch operation is provided by JAXA and NAOJ (Japan), STFC (U.K.), NASA, ESA, and NSC (Norway).

\section{References}

1) Kosugi, T., Matsuzaki, K., Sakao, T., Shimizu, T., Sone, Y., Tachikawa, S., Hashimoto, T., Minesugi, K., Ohnishi, A., Yamada, T., Tsuneta, S., Hara, H., Ichimoto, K., Suematsu, Y., Shimojo, M., Watanabe, T., Shimada, S., Davis, J. M., Hill, L. D., Owens, J. K., Title, A. M., Culhane, J. L., Harra, L. K., Doschek, G. A. and Golub, L.: The Hinode (Solar-B) Mission: An Overview, .Solar Phys., 243 (2008), pp. 5-19.

2) Tsuneta, S., Ichimoto, K., Katsukawa, Y., Nagata, S., Otsubo, M., Shimizu, T., Suematsu, Y., Nakagiri, M., Noguchi, M., Tarbell, T., Title, A., Shine, R., Rosenberg, W., Hoffmann, C., Jurcevich, B., Kushner, G., Levay, M., Lites, B., Elmore, D., Matsushita, T., Kawaguchi, N., Saito, H., Mikami, I., Hill, L. D. and Owens, J. K.: The Solar Optical Telescope for the Hinode Mission: An Overview, Solar Phys., 243 (2008), pp. 113-142, and references therein.

3) Golub, L., DeLuca, E., Austin, G., Bookbinder, J., Caldwell, D., Cheimets, P., Cirtain, J., Cosmo, M., Reid, P., Sette, A., Weber, M., Sakao, T., Kano, R., Shibasaki, K., Hara, H., Tsuneta, S., Kumagai, K., Tamura, T., Shimojo, M., McCracken, J., Carpenter, J., Haight, H., Siler, R., Wright, E., Tucher, J., Rutledge, H., Bargera, M., Peres, G. and Varisco, S.: The X-Ray Telescope (XRT) for the Hinode Mission, Solar Phys. 243 (2008), pp. 27-50.

4) Kano, R., Sakao, T., Hara, H., Tsuneta, S., Matsuzaki, K., Kumagai, K., Shimojo, M., Minesugi, K., Shibasaki, K., DeLuca, E. E., Golub, L., Bookbinder, J., Caldwell, D., Cheimets, P., Cirtain, J., Dennis, E., Kent, T. and Weber, M.: The Hinode X-Ray Telescope (XRT): Camera Design, Performance and Operations, Solar Phys., 243 (2008), pp. 51-67. 
5) Culhane, J. L., Harra, L. K., James, A. M., Al-Janabi, K., Bradley, L. J., Chaudry, R. A., Rees, K., Tandy, J. A., Thomas, P., Whillock, M. C. R., Winter, B., Doschek, G. A., Korendyke, C. M., Brown, C. M., Myers, S., Mariska, J., Seely, J., Land, J., Kent, B. J., Shaughnessy, B. M., Young, P. R., Simnett, G. M., Castelli, C. M., Mahmoud, S., Mapson-Menard, H., Probyn, B. J., Thomas, R. J., Davila, J., Dere, K., Windt, D., Shea, J., Hagood, R., Moye, R., Hara, H., Watanabe, T., Matsuzaki, K., Kosugi, T., Hansteen, V. and Wikstol, O.: The EUV Imaging Spectrometer for Hinode, Solar Phys. 243 (2008), pp. 69-111.

6) Cirtain, J. W., Golub, L., Lundquist, L., van Ballegooijen, A., Savcheva, A., Shimojo, M., DeLuca, E., Tsuneta, S., Sakao, T., Reeves, K., Weber, M., Kano, R., Narukage, N. and Shibasaki, K.: Evidence for Alfven Waves in Solar X-ray Jets, Science, 318 (2007), pp. 1580-1582.

7) Shimojo, M., Narukage, N., Kano, R., Sakao, T., Tsuneta, S., Shibasaki, K., Cirtain, J. W., Lundquist, L. L., Reeves, K. K. and Savcheva, A.: Fine Structures of Jets in the Sun's South Polar Corona, Publ. Astron. Soc. Japan, 59 (2007), pp. S745-S750.

8) Savcheva, A., Cirtain, J., DeLuca, E. E., Lundquist, L. L., Golub, L., Weber, M., Shimojo, M., Shibasaki, K., Sakao, T., Narukage, N., Tsuneta, S. and Kano, R.: A Study of Polar Jet Parameters Based on Hinode XRT Observations, Publ. Astron. Soc. Japan, 59 (2007), pp. 771-S778.

9) Tsuneta, S., Ichimoto, K., Katsukawa, Y., Lites, B. W., Matsuzaki, K., Nagata, S., Orozco Suarez, D., Shimizu, T., Shimojo, M., Shine, R. A., Suematsu, Y., Suzuki, T. K., Tabell, T. D. and Title, A. M.: The Magnetic Landscape of the Sun's Polar Region, Astrophys. J., 688 (2008), pp. 1374-1381.

10) McComas, D. J., Bame, S. J., Barraclough, B. L., Feldman, W. C., Funsten, H. O., Gosling, J. T., Riley, P., Skoug, R., Balogh, A., Forsyth, R., Goldstein, B. E. and Neugebauer, M.: Ulysses' return to the slow solar wind, Geo. Res. Lett., 25 (1998), pp. 1-4.

11) McComas, D. J., Barraclough, B. L., Funsten, H. O., Gosling, J. T., Santiago-Munoz, E., Skoug, R. M., Goldstein, B. E., Neugebauer, M., Riley, P. and Balogh, A.: Solar wind observations over Ulysses' first full polar orbit, J. Geo. Res., 105 (A5) (2000), pp. 10419-10433.

12) Moore, R. L., Musielak, Z. E., Suess, S. T. and An, C. -H.: Alfven Wave Trapping, Network Microflaring, and Heating in Solar Coronal Holes, Astrophys. J., 378 (1991), pp. 347-359.

13) Suzuki, T. K. and Inutsuka, S.: Solar winds driven by nonlinear low-frequency Alfven waves from the photosphere: Parametric study for fast/slow winds and disappearance of solar winds, J. Geo. Res., 111, A06101 (2006), pp. 1-21.

14) Shimojo, M. and Tsuneta, S., to be submitted (2009).

15) Shibata, K., Nakamura, T., Matsumoto, T., Otsuji, K., Okamoto, T. J., Nishizuka, N., Kawate, T., Watanabe, H., Nagata, S., UeNo, S., Kitai, R., Nozawa, S., Tsuneta, S., Suematsu, Y., Ichimoto, K., Shimizu, T., Katsukawa, Y., Tarbell, T. D., Berger, T. E., Lites, B. W., Shine, R. A. and Title, A. M.: Chromospheric Anemone Jets as Evidence of Ubiquitous Reconnection, Science 318 (2007), pp. 1591-1594.

16) Ishikawa, R., Tsuneta, S., Ichimoto, K., Isobe, H., Katsukawa, Y., Lites, B. W., Nagata, S., Shine, R. A., Suematsu, Y., Tarbell, T. D. and Title, A. M.: Transient horizontal magnetic fields in solar plage regions, Astron. Astrophys., 481 (2008), pp. L25-L28.

17) Ishikawa, R. and Tsuneta, S.: Comparison of transient horizontal magnetic fields in a plage region and in the quiet Sun, Astron. Astrophys., 495 (2009), pp. 607-612.

18) Isobe, H., Proctor, M. R. E. and Weiss, N. O.: Convection-Driven Emergence of Small-Scale Magnetic Fields and their Role in Coronal Heating and Solar Wind Acceleration, Astrophys. J. Lett., 679 (2008), pp. L57-L60. 